EPJ Web of Conferences 21, 10009 (2012)

DOI: $10.1051 /$ epjconf/20122110009

(C) Owned by the authors, published by EDP Sciences, 2012

\title{
How does incomplete fusion show up at slightly above barrier energies ?
}

\author{
Pushpendra P. Singh ${ }^{1,2, a}$, Abhishek Yadav ${ }^{3}$, Vijay R. Sharma ${ }^{3}$, R. Kumar ${ }^{4}$, B. P. Singh ${ }^{3}$, R. K. Bhowmik ${ }^{4, b}$, R. Prasad $^{3}$, \\ and the AMU-IUAC collaboration \\ 1 GSI Helmholtz Centre for Heavy Ion Research GmbH, D-64291 Darmstadt, Germany \\ 2 Institute for Nuclear Physics, Technical University Darmstadt, D-64289 Darmstadt, Germany \\ 3 Acc. Lab., Department of Physics, A. M. University, Aligarh - 202 002, India \\ 4 NP-Group, Inter-University Accelerator Center, New Delhi - 110 067, India
}

\begin{abstract}
Experimental results on the onset of incomplete fusion at slightly above barrier energies are discussed in this paper. Spin-distributions of evaporation residues populated via complete and/or incomplete fusion of ${ }^{12} \mathrm{C},{ }^{16} \mathrm{O}\left(\mathrm{E}_{\text {lab }} \approx 4-7 \mathrm{MeV}\right)$ with ${ }^{169} \mathrm{Tm}$ have been measured to probe associated $\ell$-values. Particle $(\mathrm{Z}=1,2)-\gamma$ - coincidence technique has been used for channel selection. Entirely different entry state spin populations have been observed during the de-excitation of complete and incomplete composites. The complete fusion residues are found to be strongly fed over a broad spin range. While, a narrow range feeding for only high spin states has been observed in case of incomplete fusion residues. In the present work, incomplete fusion is shown to be a promising tool to populate high spin states in final reaction products. For better insight into the onset and strength of incomplete fusion, the relative contributions of complete and incomplete fusion have been deduced from the analysis of excitation functions and forward recoil ranges. A significant fraction of ICF has been observed even at energy as low as $\approx 7 \%$ above the barrier. The relative strengths of complete and incomplete fusion deduced from the analysis of forward-recoil-ranges and excitation functions complement each other. All the available results are discussed in light of the Morgenstern's mass-asymmetry systematics. Incomplete fusion fraction is found to be large for more mass-asymmetric systems for individual projectiles, which points towards the projectile structure effect on incomplete fusion fraction. Experimentally measured forward ranges of recoils complement the existence of incomplete fusion at slightly above barrier energies, where more than one linear-momentum-transfer components associated with full- and/or partial-fusion of projectile(s) have been observed. Present results conclusively demonstrate the possibility to selectively populate high spin states via incomplete fusion.
\end{abstract}

\section{Introduction}

In heavy-ion induced nuclear reactions, an outstanding question is $\rightarrow$ how does incomplete fusion (ICF) show up at slightly above barrier energies ? The study of ICF at low incident energies (i.e., $\approx 4-7 \mathrm{MeV} /$ nucleon) has got resurgent interest in recent years and intensively investigated using light heavy ion $(\mathrm{A} \leq 20)$ beams [1-11]. At these energies, complete fusion (CF) is supposed to be the sole contributor to the reaction cross-section. However, a significant fraction of ICF has been observed at near barrier energies [12-18]. In general, the interaction trajectories lying within the dimensions of target nucleus prominently lead to the reaction modes namely: $(i) \mathrm{CF} \rightarrow$ defined as the capture of entire projectile by target nucleus, and (ii) ICF $\rightarrow$ where only a part of projectile fuses with target nucleus. Qualitatively, both reaction modes can be disentangled on the basis of driving angular momenta imparted into the system by the means of available interaction trajectories ( $\ell$-bins) and/or incident energy [19-22]. In general, central and/or near-central trajectories $(0 \leq \ell \leq \ell$ crit $)$ lead to $\mathrm{CF}$, where an excited composite system forms after intimate contact and transient amalgamation of projectile and target nucleus. In this case, the attractive nuclear poten-

\footnotetext{
a e-mail: pushpendrapsingh@gmail.com

b Present Address: Department of Pure and Applied Physics, Guru Ghasidas University, Koni, Bilaspur - 495009 (CG), India
}

tial influences the sum of repulsive Coulomb and centrifugal potentials. Eventually, the projectile's total kinetic energy and angular momenta are equally distributed among all the accessible internal degrees of freedom of composite system to form an equilibrated compound nucleus (eq$\mathrm{CN})$. However, at relatively higher $\ell$-values $\left(\geq \ell_{\text {crit }}\right)$, the centrifugal potential overwhelms attractive nuclear potential, therefore, the pocket in the entrance channel potential disappears that hinders the fusion of entire projectile (CF) with target nucleus and gives way to partial fusion (ICF). In this case, projectile releases excess driving angular momenta by emitting an $\alpha$-cluster(s) or a group of nucleons as spectator $\left(\mathrm{P}^{s}\right)$. After such an emission, the remnant (termed as participant: $\mathrm{P}^{p}$ ) supposed to have an effective driving angular momenta $\left(\ell_{e f f}\right)$ less than or equals to its own critical limit $\left(\ell_{e f f} \leq \ell_{c r i t}^{P^{p}+T}\right)$ for fusion to occur. Fusion of projectile remnant $\left(\mathrm{P}^{p}\right)$ with target nucleus $\left(\mathrm{A}_{T}\right)$ leads to an incompletely fused composite (IFC $\rightarrow \mathrm{CN}^{\star}=$ $\mathrm{P}^{p}+\mathrm{A}_{T}$ ), and direct projectile-like-fragments (PLFs $\rightarrow$ $\left.\mathrm{P}^{s}\right)$ mainly concentrated in the forward cone. As a matter fact, the eq-CN formed via $\mathrm{CF}$ process is expected to be of pre-determined mass/charge, excitation energy and angular-momenta. However, the incompletely fused composite system $\left(\mathrm{CN}^{\prime}\right)$ forms with relatively less mass/charge and excitation energy (due to partial fusion of projectile), but at high angular-momenta (imparted into the system

This is an Open Access article distributed under the terms of the Creative Commons Attribution-Noncommercial License 3.0, which permits unrestricted use, distribution, and reproduction in any noncommercial medium, provided the original work is properly cited. 
due to non-central interactions) as compared to the eq-CN formed via $\mathrm{CF}$ process.

Since the very first experimental observations of directPLFs by Britt and Quinton [23] and Galin et al.[24], there has been great interest in the dynamics of PLFs production termed as ICF. Several dynamical models have been proposed to explain the dynamics of PLFs production. In SUMRULE model, Wilczynski et al.[20-22], suggested that the process of ICF is mainly confined to the $\ell$-space above $\ell_{\text {crit }}$ for $\mathrm{CF}$, and originates from peripheral interactions or non-central collisions. The non-central nature of ICF has also been emphasized by Trautmann et al. [25], and Inamura et al.[26-28]. The break-up fusion (BUF)-model [29] of Udagawa and Tamura is based on the Distorted Wave Born Approximation ( $D W B A$ ), in which the projectile is assumed to break-up into constituent $\alpha$-clusters (e.g., ${ }^{16} \mathrm{O}$ may break-up into ${ }^{12} \mathrm{C}+\alpha$ and/or ${ }^{8} \mathrm{Be}+{ }^{8} \mathrm{Be}$ ) within the nuclear field of target nucleus. One of the fragments may get fuse with target nucleus and remnant behaves like a spectator ejected in the forward cone. According to promptly emitted particles (PEP)-model [30], the nucleons transferred from projectile to the target nucleus may get accelerated in the nuclear field of target nucleus and consequently acquire enough extra velocity to escape before equilibration of the composite system. Further, Morgenstern et al. [31-33], correlated the onset of ICF with relative velocity $\left(v_{\text {rel }}\right)$ where ICF is found to be contributed significantly above $v_{r e l} \approx 0.06$ (6\% of c). In an outstanding review, Gerschel [34] inferred that the localization of $\ell$-window also depends on the target deformation. Despite a variety of existing studies (see refs. [4,5,9] for more details), the dynamics of ICF at low incident energies is still not fairly understood, thus continues to be an active area of investigations. In the present scenario, the most debated issues related to ICF are; $(i)$ the localization of $\ell$-values, (ii) the onset of ICF at slightly above barrier energies, and (iii) the projectile and/or mass-asymmetry effect on ICF fraction.

For an insight into the aforementioned issues related to ICF dynamics, high quality data have been obtained in a variety of experiments performed at the Inter-University Accelerator Center (IUAC), New Delhi using ${ }^{12} \mathrm{C},{ }^{16} \mathrm{O}$ beams delivered from 15UD - Tandem accelerator [1-6, $10,11]$. Some of the interesting results along with a short account of experimental conditions are summarized in this paper. In first set of experiments, the spin (J)-distributions of evaporation residues have been measured using well established particle- $\gamma$-coincidence technique to take advantage of its high degrees of reaction channel selectivity [1$3,10,11]$. The experimental conditions and some of the important findings of spin-distribution measurement are briefly discussed in section-2. It may be pointed out that the absolute production production cross-sections of reaction residues could not be measured in the spin-distribution measurement. Therefore, two complementary experiments have been performed to measure ICF strength function [4, $6,10,11]$, and mean forward-recoil-ranges (FRRs) [5, 10, 11] using novel activation technique. A new approach to deduce ICF strength function from the analysis of experimental excitation functions in terms of eq-CN decay is given in section-3. While, the validation of this approach is discussed in section-4, and the summary of the present work is given in the last section of this paper.

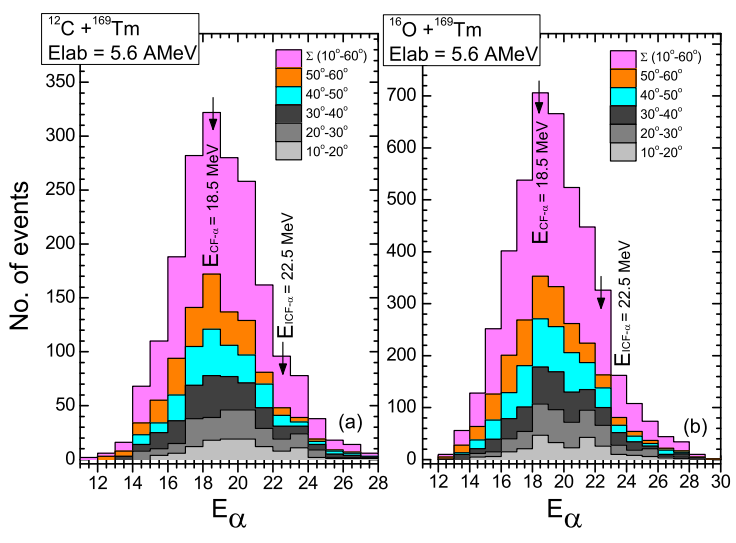

Fig. 1. (a) Fusion-evaporation (CF) $\alpha$-energy profile for for$\operatorname{ward}(\mathrm{F})$ angular zone $\left(10^{\circ}-60^{\circ}\right)$ obtained at energy $\mathrm{E}_{l a b}=5.6$ $\mathrm{AMeV}$ for ${ }^{12} \mathrm{C},{ }^{16} \mathrm{O}+{ }^{169} \mathrm{Tm}$ systems using statistical model code PACE4. Distribution of $\mathrm{CN}_{\alpha-C F}$ in different angular slices from $10^{\circ}$ to $60^{\circ}$, and the expected energy of fusion-evaporation $\alpha$ particles $\left(\mathrm{E}_{\alpha-C F}=18.5 \mathrm{MeV}\right)$ and direct $\alpha$-particles $\left(\mathrm{E}_{\alpha-I C F}=\right.$ $22.5 \mathrm{MeV}$ ) emitted in ICF processes are also shown.

\section{Experimental details and off-line data analysis procedure}

First set of experiments has been performed to probe the role of high $\ell$-values in the onset of ICF at slightly above barrier energies, where CF is supposed to be the sole contributor. The spin-distributions (SDs) of $\mathrm{xn} / \mathrm{pxn} / \alpha \mathrm{xn} / 2 \alpha \mathrm{xn}$-channels have been measured in ${ }^{12} \mathrm{C},{ }^{16} \mathrm{O}+{ }^{169} \mathrm{Tm}$ systems at energies $\approx 4-7 \mathrm{MeV} /$ nucleon [1-3]. The particles $(Z=1,2)$-xoincidences have been recorded for the identification of reaction residues using Gamma Detector Array (GDA) and Charged Particle Detectors (CPDs) Array setups. The GDA is an assembly of 12 Compton suppressed, high resolution HPGe-detectors installed in three rings at angles $45^{\circ}, 99^{\circ}, 153^{\circ}$ with respect to the beam axis and there are 4 detectors in each of these rings. The CPDs-array is a set of 14-phoswich detectors housed in a 14-cm diameter scattering chamber, covering $\approx 90 \%$ of the total solid angle. In order to get angulardistributions of charged particles, all 14 detectors of CPDsarray have been divided into the three angular zones; $(i)$ Forward-(F) $10^{\circ}-60^{\circ}$, (ii) Sideways-(S) $60^{\circ}-120^{\circ}$ and (iii) Backward-(B) $120^{\circ}-170^{\circ}$. The coincidences were demanded between particles $(Z=1,2)$ and prompt- $\gamma$-rays by employing three gating conditions corresponding to given angular zones for each value of $\mathrm{Z}$. Depending on the fast and slow components of the CPDs, particles (a sum of protons and $\alpha$ 's) and $\alpha$ 's in each angular zone have been detected in coincidence with prompt- $\gamma$-rays. As a matter of fact, the CPDs at forward (F) angles $\left(10^{\circ}-60^{\circ}\right)$ are expected to detect both slow and fast $\alpha$-components, i.e., $(i)$ slow $\alpha$-component: fusion-evaporation (CF) $\alpha$-particles, and (ii) fast $\alpha$-component: direct $\alpha$-particles associated with ICF. In order to record only fast $\alpha$-component (associated with ICF) in forward cone, its essential to stop slow $\alpha$-component in forward(F) cone by putting an absorber onto the forward CPDs. In order to know the absorber's thickness, the energy profile of slow $\alpha$-particles 
(emitted from fully eq-CN) has been generated by theoretical model code PACE4 [35] (see Fig.1). This code is based on statistical approach of $\mathrm{CN}$ de-excitation by Monte Carlo procedure, and has extensively been used for $\mathrm{CN}$ related calculations in past years. Similar input parameters, as in ref.[3], has been used for this calculation. As can be seen in Fig.1(a), the theoretically estimated most probable energy of fusion-evaporation $\alpha$-particles $\left(\mathrm{E}_{C F-\alpha}\right)$ is found to be $\approx$ $18.5 \mathrm{MeV}$ at $\approx 5.6 \mathrm{AMeV}$. However, as per definition of $\mathrm{ICF}$, the energy of fast $\alpha$-component $\left(\mathrm{E}_{I C F-\alpha}\right)$ can be calculated as projectile energy times ejectile-projectile mass ratio, and comes out to be $\approx 22.5 \mathrm{MeV}$ at $67.5 \mathrm{MeV}$ for ${ }^{12} \mathrm{C}$-beam. As such, to stop $\approx 18.5 \mathrm{MeV}$ slow $\alpha$-particles, an Al-absorber of appropriate thickness was kept onto the forward (F) CPDs so that only fast- $\alpha$-component in forward cone can be detected. In-beam prompt $\gamma$-ray spectra have been recorded in event-by-event multi parameter mode, which includes different coincidences like; $\alpha$ and $2 \alpha$ detected in backward (B), forward (F) and $90^{\circ}(\mathrm{S})$ - angular zones. Singles data have also been collected to identify xn-channels (produced via $\mathrm{CF}$ ).

Off-line data analysis has been performed in two steps. In first step, the xn-channels have been identified by looking into singles data. For the identification of pxn-channels, backward(B)- $\alpha$-gated $\gamma$-spectra has been subtracted from backward(B)-particles $(Z=1,2)$-gated $\gamma$-spectra to achieve proton(p) gated $\gamma$-spectra. However, $\alpha \times n / 2 \alpha \times n(\mathrm{CN}-\alpha)$-channels produced via $\mathrm{CF}$ have been identified from the backward(B)- $\alpha$-gate $\alpha$-spectra. As per the justified definition of ICF, the direct- $\quad \alpha$-particles (PLFs) are expected to be concentrated in the forward- $(\mathrm{F})$ angular zone. As such, $\alpha \mathrm{xn} / 2 \alpha \mathrm{xn}($ direct- $\alpha)$-channels produced via ICF have been identified from the forward(F)- $\alpha$-gated $\gamma$-spectra. The intensity and area under the peaks (efficiency corrected) of the characteristic prompt- $\gamma$-transitions assigned to a particular reaction product was used to determine the relative production yield.

\subsection{Spin (J)-distributions: hints of reaction dependent entry state spin population}

The fact that the relative number of statistical and 'yrast'like transitions depend on entry state angular-momenta and available excitation energy $\left(\mathrm{E}^{*}\right)$. The $\mathrm{CF}$ reaction products are supposed to be formed at high $\mathrm{E}^{*}$ and low $\ell$-values leading to more statistical transitions, where 'yrast' states are expected to fed by statistical $\gamma$-transitions. However, the ICF reaction products achieve relatively low $\mathrm{E}^{*}$ (due to the involvement of partial degrees of excitations) and high angular-momenta (relatively higher values of impact parameters contribute to the high spin states) at a given projectile energy. In this case, the number of 'yrast'-like transitions are much higher than that of statistical ones, where less or no feeding is expected. Therefore, the spindistributions of $\mathrm{CF}$ and ICF products are expected to be entirely different in nature and can be used as a sensitive tool to probe reaction dynamics by looking into entry state spin-population(s). In order to generate experimental spindistributions of $\mathrm{CF}$ and ICF residues, relative production yields have been plotted as a function of experimentally observed spin $\left(\mathbf{J}_{o b s}^{e x p}\right)$ corresponding to prompt $\gamma$-transitions. For better comparison of $\mathrm{xn}, \alpha \mathrm{xn}$ and $2 \alpha \mathrm{xn}$ - channels in a panel, relative production yields have been normalized

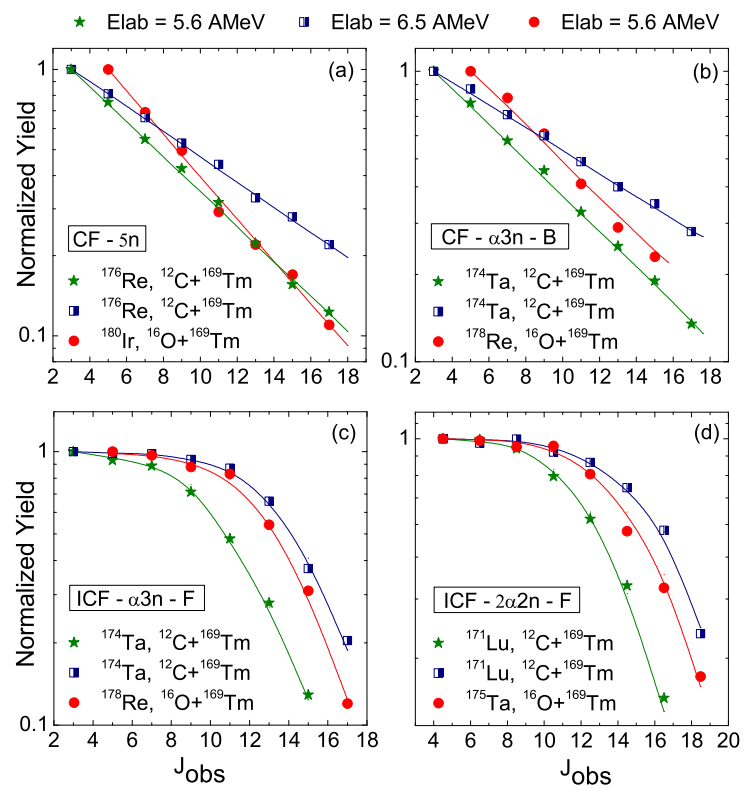

Fig. 2. Experimentally measured spin-distributions of (a) $5 n$ (b) $\alpha 3 n-B$ (c) $\alpha 3 n-F$, and (d) $2 \alpha 2 n-F$ channels populated in ${ }^{12} \mathrm{C},{ }^{16} \mathrm{O}+{ }^{169} \mathrm{Tm}$ systems. Different reaction products have been labeled by self-explanatory notations and emission cascades. 'B' and ' $\mathrm{F}$ ' symbols respectively represent the residues identified from backward (B)- $\alpha-\gamma$-coincidences and forward $(F)-\alpha-\gamma-$ coincidences. The lines and curves drawn through the data points are the result of best fit procedure explained in the text.

with their own highest measured values $\left(\mathrm{Y}^{\max }\right)$ at lowest observed spin $\mathrm{J}_{o b s}^{\mathrm{min}}$.

Further, as an analytical representation of data, the experimentally measured spin-distributions obtained as mentioned above have been fitted by a function of following type;

$$
Y=Y_{\circ} /\left[1+\exp \left(J-J_{\circ}\right) / \Delta\right]
$$

Here; $\Delta$ is related to the width of mean input-angular momenta $\left(J_{\circ}\right)$ and $Y_{\circ}$ is the normalization constant. $J_{\circ}$ is a sensitive parameter, which provides the qualitative information about the driving input angular-momenta associated with various reaction channels.

The spin-distributions of $x \mathrm{n} / \mathrm{p} x \mathrm{n} / \alpha x \mathrm{n} / 2 \alpha x \mathrm{n}$ channels expected to be populated via $\mathrm{CF}$ and/or ICF in ${ }^{12} \mathrm{C},{ }^{16} \mathrm{O}+{ }^{169} \mathrm{Tm}$ systems are plotted in Fig.2(a-d) as a representative case. The errors are not plotted in the figures as they have been estimated to be $\leq 10 \%$, and the inclusion of these errors do not modify the present analysis. The nomenclature used in this figure indicate the involved reaction dynamics, i.e., ' $\mathrm{B}$ ' and ' $\mathrm{F}$ ' respectively indicate CF-channel: identified from backward(B)- $\alpha$-gated spectra, and ICF-channel: identified from forward(F)- $\alpha$-gated spectra. As can be seen from this figure, there is a striking difference in the spin-distributions of $\mathrm{xn} / \alpha \mathrm{xn} / 2 \alpha \mathrm{xn}$-channels. This indicates the involvement of different reaction dynamics in the production of these residues. For an example, as shown in Fig.2(a)-(b), the intensity of CF- 5 n(singles) $/ \alpha 3 n$ $\mathrm{B}$ (identified from backward- $\alpha$-gated $y$-spectra) falls off rather quickly with observed spin $\left(\mathrm{J}_{o b s}\right)$, indicating strong 

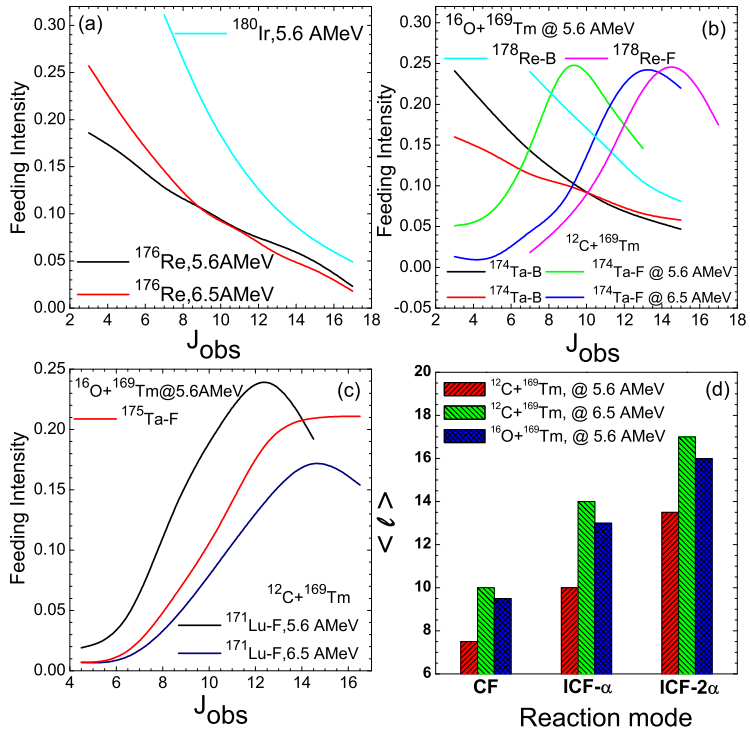

Fig. 3. Feeding intensity profiles of $\gamma$-cascades of different reaction products populated via, (a) CF-xn (identified from singles-spectra), ( $b$ ) $\mathrm{CF} / \mathrm{ICF}-\alpha \mathrm{xn}$ (identified from backward(B)/forward(F)- $\alpha$-gated spectra), and (c) ICF- $2 \alpha \times$ n (identified from forward(F)- $\alpha$-gated spectra) channels deduced from experimentally measured spin-distributions. Lines and curves are obtained from the best fit of experimental data points, (d) The value of mean driving angular momenta (i.e., the value of $\mathrm{J}_{\circ}$ deduced from the best fitting procedure of spin-distributions as explained in ref.[?]) involved in various $\mathrm{CF}$ and ICF channels as a function of reaction modes.

feeding towards band head and/or broad spin-population during the de-excitation of $\mathrm{CN}$. However, distinctly different decay patterns are found in case of ICF- $\alpha 3 n-F$ (identified from forward- $\alpha$-gate $\alpha$-spectra) and CF- $\alpha 3 n$-B (identified from backward- $\alpha$-gated $y$-spectra). In case of ICF$\alpha 3 n-F$ and ICF- $2 \alpha 2 n-F$, refer to Fig.2(c)-(d), the intensity appears to be almost constant up to a certain value of $\mathrm{J}_{o b s}$, and then decreases towards entry side. This indicates the absence of feeding to the lowest members of the 'yrast' band and/or the population of low spin states are strongly hindered in ICF channels. In order to get better insights into the mean $\ell$-values and feeding probability involved in case of $\mathrm{CF}$ and ICF products, channel dependent $\ell$-correlations and feeding intensity profiles (FIPs) have been generated from the analysis of experimental spin-distributions. The FIPs and channel dependent $\ell$-correlations are given in the following sections.

\subsection{Feeding intensity profiles: indication of narrow range spin-populations in ICF}

The FIPs of different CF and ICF products are plotted in Fig.3(a-c). As can be seen from Fig.3a, the feeding intensity for CF-5n-channel shows sharp exponential rise towards the low spin states, which indicates continuous wide range spin-population with strong feeding contribution for each $\gamma$-transition up to $\mathrm{J}_{o b s}^{\min }$. This feature can be better understood if an ICF channel is compared with the
CF channel in the same panel. Fig.3(b) shows the comparison of FIPs for direct- $\alpha$-emitting (ICF- $\alpha 3 n-F)$ and fusionevaporation $\alpha$-emitting (CF- $\alpha 3$ n-B) channels. The feeding intensity of CF- $\alpha 3 \mathrm{n}$ channel identified from backward(B)$\alpha$-gated spectra shows almost similar trend as that has been observed for CF-5n channel, where the band is fed over a broad spin range. While, the feeding intensity for ICF$\alpha 3 \mathrm{n}-\mathrm{F}$ channel (identified from forward- $\alpha$-gated spectra) is found to be increasing up to a certain values of $\mathrm{J}_{o b s}$ and then decreases gradually towards the band head. This trend of ICF- $\alpha 3 \mathrm{n}-\mathrm{F}$ channel indicates that the high spin states are strongly fed even in case of ICF. However, as the residual nucleus de-excites, the feeding intensity decreases gradually with available excitation energy and/or angular momenta. This clearly shows the absence of feeding to the lowest members of the 'yrast' band in case of ICF, or the low spin states are less populated in ICF-channels. Such feeding intensity pattern is expected to arise from narrow $\ell$-window (or narrow range spin-populations) localized near and/or above the critical angular momentum for CF. Similar trend has also been observed in ICF- $2 \alpha 2 n-F$ channel (see Fig.3c). Detailed discussion on FIPs of different $\mathrm{CF} / \mathrm{ICF}$ - channels can be found in our recent papers [1-3].

\subsection{Associated $\ell$-values and the possibility to populate high spin states via ICF}

In order to figure out the multitude of $\ell$-values involved in different reaction channels, and to examine the possibility to populate high spin states via ICF, the mean $\ell$-values $(<\ell>)$ involved in CF and ICF channels have been deduced from the analysis of spin-distributions. As discussed in the previous section, the value of $J_{\circ}$ in the Fermi function provides the mean $\ell$-values associated with various reaction channels. The mean $\ell$-values obtained from the best fitting procedure of experimental spin-distributions are plotted in Fig.3(d). For an example, refer to Fig.3d, the value of $\langle\ell\rangle$ involved in the production of $\mathrm{CF}-\mathrm{xn} / \mathrm{pxn}$ $\mathrm{B} / \alpha \mathrm{xn}-\mathrm{B}, \mathrm{ICF}-\alpha \mathrm{xn}-\mathrm{F}$, and ICF- $2 \alpha \times \mathrm{xn}-\mathrm{F}$ channels are found to be $\approx 7.5 \hbar, \approx 10 \hbar$ and $\approx 13.5 \hbar$, respectively, at projectile energy $\approx 5.6 \mathrm{AMeV}$ for ${ }^{12} \mathrm{C}+{ }^{169} \mathrm{Tm}$ system. However, at projectile energy $\approx 6.5 \mathrm{AMeV}$ for the same projectiletarget combination, the value of $\langle\ell\rangle$ for CF-xn/pxn$\mathrm{B} / \alpha \mathrm{xn}-\mathrm{B}, \mathrm{ICF}-\alpha \mathrm{xn}-\mathrm{F}$, and ICF- $2 \alpha \mathrm{xn}-\mathrm{F}$ channels are found to be $\approx 10 \hbar, \approx 14 \hbar$ and $\approx 17 \hbar$, respectively. The enhancement in the value of $\langle\ell\rangle$ in case of direct- $\alpha$-emitting channels (ICF products) indicates their origin from high $\ell$-values as compared to $\mathrm{CF}$-channels. A very useful correlation between the value of $\langle\ell\rangle$ and the successively opened ICF channels can be obtained from the data presented in Fig.3(d). Following the approach presented in our recent letter [1], the value of $\langle\ell\rangle$ associated with ICF in contrast with $\mathrm{CF}$ can be represented as;

$$
\begin{aligned}
& \text { (i) for }{ }^{12} \mathrm{C}+{ }^{169} \mathrm{Tm} \text { system at } \mathrm{E}_{l a b} \approx 5.6 \mathrm{AMeV} ; \\
& \ell_{(I C F-\alpha x n)} \approx 1.33 \ell_{(C F-x n / p x n / \alpha x n)}, \\
& \ell_{(I C F-2 \alpha x n)} \approx 1.35 \ell_{(I C F-\alpha x n)} \approx 1.8 \ell_{(C F-x n / \alpha x n)}, \text { and } \\
& \text { (ii) at } \mathrm{E}_{l a b} \approx 6.5 \mathrm{AMeV} ; \\
& \ell_{(I C F-\alpha x n)} \approx 1.4 \ell_{(C F-x n / p x n / \alpha x n)}, \\
& \ell_{(I C F-2 \alpha x n)} \approx 1.2 \ell_{(I C F-\alpha x n)} \approx 1.7 \ell_{(C F-x n / \alpha x n)}
\end{aligned}
$$


It is interesting to note that the values of $\langle\ell>$ involved in the production of various ICF- $\alpha \times \mathrm{xn} / 2 \alpha \mathrm{xn}$-channels are found to be $\approx 30$ to $70 \%$ higher as compared to the $\mathrm{CF}-\mathrm{xn} / \mathrm{pxn} / \alpha \mathrm{xn}$-channels at both the energies. This indicates the involvement of high $\ell$-values in the production of ICF products at a constant incident energy. This also confirms the origin of ICF events from the non-central interactions, where a significant amount of orbital angular momentum between projectile and target nucleus transformed into high spin states of final reaction products.

Further, concerning the usefulness of ICF as a tool to populate high-spin states, it can be noted down from Fig.2(b)-(c) that the same residue ${ }^{174} \mathrm{Ta}(\alpha 3 \mathrm{n})$ can be populated at high spin states via ICF as compared to CF. As a more precise example, as can be seen from Fig.2(b)-(c), the value of $\langle\ell\rangle$ at $\approx 5.6 \mathrm{AMeV}$ is found to be $\approx 7.5 \hbar$ involved in the production of ${ }^{174} \mathrm{Ta}(\alpha 3 \mathrm{n}-\mathrm{B})$ identified from backward(B)- $\alpha$-gated spectra (associated with CF). However, if the same residue (at same projectile energy) identified from forward(F)- $\alpha$-gated spectra, which supposed to be populated via ICF, the value $\langle\ell\rangle$ is found to be $\approx 10.5$ $\hbar$ (refer to Fig.2c). Apart from that, the value of $\langle\ell>$ associated with the production of ${ }^{174} \mathrm{Ta}$ via $\mathrm{CF}$ at $\approx 6.5 \mathrm{AMeV}$ (i.e., $\approx 9.5 \hbar$ ) is achieved via ICF even at lower projectile energy $\approx 5.6 \mathrm{AMeV}$ (i.e., $\approx 10.5 \hbar$ ). Similar characteristics have been observed in case of other reaction channels populated via both CF and ICF. For better representation, the above information on $\langle\ell>$-values involved in different reaction channels can be correlated as;

$$
\begin{aligned}
& \text { (i) for }{ }^{174} \mathrm{Ta}(\alpha 3 \mathrm{n}) ; \\
& \ell_{(I C F-\alpha 3 n)} \approx 1.33 \ell_{(C F-\alpha 3 n)}, \text { at } \mathrm{E}_{l a b} \approx 5.6 \mathrm{AMeV} \text {, and } \\
& \ell_{(I C F-\alpha 3 n)} \approx 1.4 \ell_{(C F-\alpha 3 n)}, \text { at } \mathrm{E}_{l a b} \approx 6.5 \mathrm{AMeV} \\
& \text { (ii) for }{ }^{174} \mathrm{Ta}(\alpha 3 \mathrm{n}) ; \\
& \ell_{(I C F-\alpha 3 n)}(\approx 5.6 \mathrm{AMeV}) \approx 1.1 \ell_{(C F-\alpha 3 n)}(\approx 6.5 \mathrm{AMeV})
\end{aligned}
$$

As discussed above, at $\approx 5.6 \mathrm{AMeV}$, ICF can populate ${ }^{174} \mathrm{Ta}(\alpha 3 \mathrm{n})$ with $\approx 33 \%$ more angular momentum as compared to that populated via $\mathrm{CF}$, and $\approx 40 \%$ more at $\approx 6.5$ $\mathrm{AMeV}$. Further, it is also clear from the second correlation that the CF is not able to populate the same amount of angular momenta even at $\approx 6.5 \mathrm{AMeV}$ which is populated via ICF in ${ }^{174} \mathrm{Ta}(\alpha 3 \mathrm{n})$ at relatively low projectile energy, i.e., $\approx 5.6 \mathrm{AMeV}$. The above striking feature strongly support the possibility to populate high spin states via ICF in final reaction products even at low bombarding energies.

Further, refere to Fig.3(d), the involved $\ell$-values in different reaction channels are found to increase linearly with the projectile energy, and almost same (within $\leq 0.5 \hbar$ ) for each set of reaction channels at a given projectile energy. The smallness of $J_{\circ}$ indicates the involvement of less input angular momenta in CF reactions. It may also be seen from the deduced values of $J_{\circ}$ that the multiplicity of $\alpha$-particles associated with ICF increases with the driving input angular momenta, indicating the variation of different $\ell$-values (clear signature of impact parameter dependent) at a given projectile energy. This indicates that the partial waves of lower $\ell$-values do not contribute to the ICF reaction significantly. Therefore, it may not be out of order to state that ICF reactions occur in the peripheral interactions, probably at finite values of impact parameters, where the centrifugal force field overtakes the sum of nuclear and Coulomb potentials.

\section{ICF strength function}

In order to achieve information on how does the fraction of ICF depend on various entrance channel parameters, the value of incomplete fusion fraction $\left(\mathrm{F}_{I C F}\right)$ has been deduced as a function of projectile energy and massasymmetry of interacting partners . In a separate set of experiments, absolute production cross-sections of individual reaction residues populated via $\mathrm{CF}$ and/or ICF in ${ }^{12} \mathrm{C},{ }^{16} \mathrm{O}+{ }^{169} \mathrm{Tm}$ systems have been measured at energies $\approx 4-7 \mathrm{MeV} /$ nucleon [4,6,9]. Experiments have been performed using General Purpose Scattering Chamber (GPSC)- setup at IUAC. The experimental setup and methodology have been detailed in refs.[4,6,9]. However, a brief account of experimental conditions is given here for ready reference. Natural ${ }^{169} \mathrm{Tm}$ (abundance $=100 \%$ ) Targets of thickness $\mathrm{t}_{m} \approx 1-1.3 \mathrm{mg}-\mathrm{cm}^{-2}$ were prepared by uniform pressure rolling (UniPRo)- technique. The uniformity of each target foil was verified by multi-point thickness measurement using $\alpha$-transmission method. For ${ }^{16} \mathrm{O}+{ }^{169} \mathrm{Tm}$ system, energy degradation technique has been employed to cover a wide energy range in one run, and the corrections were made for energy spread and beam intensity variations. While, in case of ${ }^{12} \mathrm{C}+{ }^{169} \mathrm{Tm}$ system, the energy spread and beam intensity variations have been avoided by adopting single target irradiation methodology for each projectile energy. Irradiations were carried out using ${ }^{12} \mathrm{C},{ }^{16} \mathrm{O}$-beams for an energy range of $\approx 4-7$ $\mathrm{MeV} /$ nucleon with a beam current $\approx 20-30 \mathrm{nA}$. All targets were backed by Al-catchers of appropriate thicknesses, so that the recoiling reaction products can be trapped in the catcher foil thickness. Proper care was taken to maintain the constant beam current during the irradiations. Measurements of $\gamma$-activities produced during the irradiations were performed off-line using two pre-calibrated HPGe detectors. The uncertainty in geometry dependent efficiency of detectors is estimated to be $\leq 2 \%$. Prompt- $\gamma$-rays have also been recorded in singles mode for an additional degree of freedom in residue identification. Reaction products have been identified by their characteristic promptand decay- $\gamma$-lines. In order to gain high confidence in the identification of reaction products, decay-curve analysis for each residue has been performed using decay- $\gamma$-lines. The most intense decay $\gamma$-lines have been used for decaycurve analysis and for the production cross-section $\left(\sigma_{E R}\right)$ measurement [3]. The projectile energy dependent reaction cross-sections $\left(\sigma_{r}(E)\right)$ for different reaction products have been determined using standard formulation as given ref.[9]. The overall error in the production cross-sections $\sigma_{E R}$ is estimated to be $\leq 13 \%$, excluding the uncertainty in branching ratio, decay constant etc. Detailed discussions on error estimation and causing factors are given elsewhere [9].

The value of $\mathrm{F}_{I C F}$ has been deduced from the analysis of experimental EFs in the framework statistical model code PACE4. Details of this code can be found in our recent papers $[4,9]$. This code is based on the hypothesis of equilibrated compound nucleus decay, and has been used extensively to characterize the fusion-evaporation component in the given mass region. In this code, the level den- 

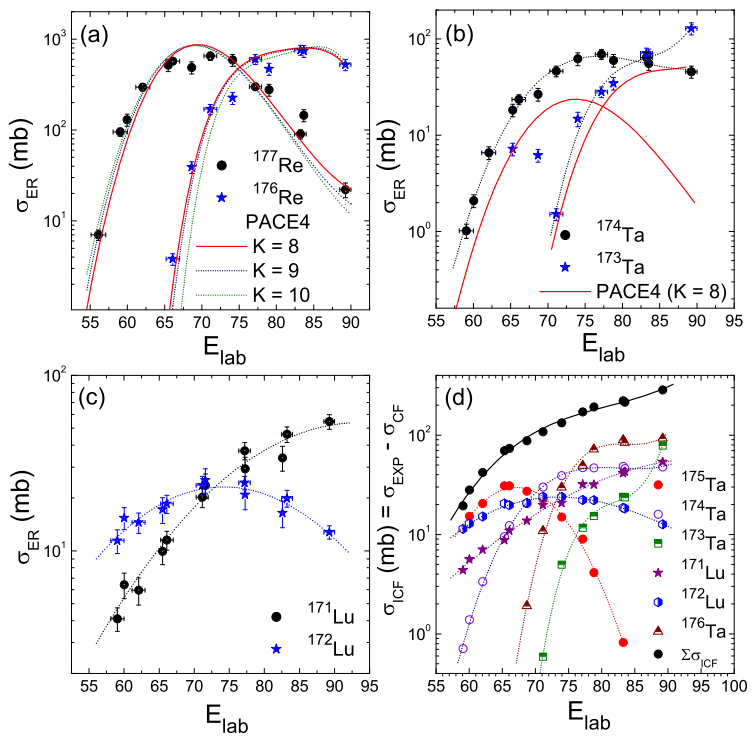

Fig. 4. Experimentally measured EFs of (a) ${ }^{176,177} \operatorname{Re}(5 n / 4 n),(b)$ ${ }^{173,174} \mathrm{Ta}(\alpha 4 \mathrm{n}, \alpha 3 \mathrm{n})$, and $(c){ }^{171,172} \mathrm{Lu}(2 \alpha 2 \mathrm{n}, 2 \alpha \mathrm{n})$ residues expected to be populated via CF and/or ICF of ${ }^{12} \mathrm{C}$ with ${ }^{169} \mathrm{Tm}$, and $(d)$ Experimentally measured and systematically deduced (see text for the deduction procedure) EFs of ICF products along with the sum of all ICF-channels $\left(\sum \sigma_{I C F}\right)$. Lines and symbols are used for self explanatory notations.

sity parameter $(\mathrm{a}=\mathrm{A} / \mathrm{K})$ is an important input parameter, which may be adjusted to fit the experimental data. In order to derive the suitable value of ' $a$ ', different values of ' $a$ ' from $\mathrm{A} / 10$ to $\mathrm{A} / 8 \mathrm{MeV}^{-1}$ have been tested in the present work. As a representative case, the EFs of ${ }^{176,177} \operatorname{Re}(5 n, 4 n)$ residues are compared with that estimated by PACE4 for different level density parameters in Fig.4(a). As can be seen from this figure, the EFs of ${ }^{176,177}$ Re residues are very well reproduced for the value of $\mathrm{a}=\mathrm{A} / 8 \mathrm{MeV}^{-1}$. This confirms the production of these residues $\left({ }^{176,177} \mathrm{Re}\right)$ through the eq-CN-decay via emission of $x$-neutrons from the excited ${ }^{185} \mathrm{Ir}^{\star}$ formed in a CF process. Therefore, the value of $\mathrm{a}=\mathrm{A} / 8 \mathrm{MeV}^{-1}$ can be used as default input parameter for the analysis of $\alpha$-emitting channels within the given energy range.

Fig.4(b) shows the EFs of ${ }^{173,174} \mathrm{Ta}(\alpha 4 \mathrm{n}, \alpha 3 \mathrm{n})$ residues expected to be populated via $\mathrm{CF}$ and/or ICF. The EFs of these residues are significantly enhanced than that predicted by statistical model code PACE4. This enhancement over the PACE4 predictions may be the indication of some physical effect which is not included in code PACE4. It may be pointed out that the code PACE4 do not take ICF into account. Therefore, the enhancement in case of $\alpha 3 \mathrm{n}$ and $\alpha 4 \mathrm{n}$ channels may be attributed to the onset of ICF. Assuming this enhancement as the contribution of ICF, the fraction of ICF has been accounted as, $\sigma_{I C F}=\sigma_{\text {exp }}$ $\sigma_{\text {pace } 4}[14,3]$. In case of $2 \alpha 2 \mathrm{n}$ and $\alpha 2$ n-channels (as shown in Fig.4c), the code PACE4 predicts negligible production cross-section. This indicates the population of these residues only via ICF. Further, Fig.4d displays the experimentally measured and systematically deduced ICF fraction $\left(\sigma_{I C F}\right)$ in the production of $\alpha$-emitting channels. Solid black line through the data points represents the sum of all ICF channels $\left(\Sigma \sigma_{I C F}\right)$. As indicated in this figure, the value of $\Sigma \sigma_{I C F}$ increases linearly with incident energies which confirms the ICF energy dependence, as expected.

\subsection{Projectile energy dependence of ICF}

In order to show how does ICF contribute to the total fusion cross-section (i.e., $\sigma_{T F}=\Sigma \sigma_{C F}+\Sigma \sigma_{I C F}$ ) ? The value of $\Sigma \sigma_{I C F}$ is plotted with the sum of all CF channels $\left(\Sigma \sigma_{C F}\right)$ and $\sigma_{T F}$ on linear scale in Fig.5(a) for an easy visualization of increasing ICF contribution with energy. The increasing separation between $\Sigma \sigma_{C F}$ and $\sigma_{T F}$ with energy shows that the ICF fraction strongly depends on incident projectile energy. For better insight into the projectile energy effect on ICF fraction, the percentage fraction of ICF $\left(\mathrm{F}_{I C F}\right)$ has been deduced from the re-analysis of data presented in Fig.5a as;

$$
F_{I C F}=\frac{\Sigma \sigma_{I C F}}{\Sigma \sigma_{C F}+\Sigma \sigma_{I C F}} \times 100
$$

The value of $\mathrm{F}_{I C F}$ is plotted as a function of projectile energy in Fig.5(b), i.e., termed as ICF strength function. The ICF strength function defines empirical probability of ICF at different incident energies. As can be seen from this figure, the value of $\mathrm{F}_{I C F}$ is found to be $\approx 7 \%$ at $\approx 59 \mathrm{MeV}$, i.e., $1.075 \mathrm{Vb}$ ( $7.5 \%$ above the barrier $)$, and increases smoothly up to $\approx 18 \%$ at highest measured energy (i.e., $1.64 \mathrm{Vb}$ ). The existence of ICF at such a low energy has been justified as a consequence of high input $\ell$-values imparted into the system in non-central interactions [1-3, 20-22].

\subsection{Mass-asymmetry or $\mathrm{Z}_{P}$ or projectile structure dependence of ICF}

Following the Morgenstern's mass-asymmetry systematics [31-33], the values of $\mathrm{F}_{I C F}$ obtained for ${ }^{12} \mathrm{C},{ }^{16} \mathrm{O}+{ }^{169} \mathrm{Tm}$ systems are plotted as a function of relative velocity $\left(v_{\text {rel }}\right.$ $\rightarrow \beta$ ) in Fig.5c. The energy axis is normalized to incorporate the Coulomb barriers of two systems, and to keep the same observable as has been used in most of the previous studies. According to Morgenstern's systematics [31-33], ICF contributes significantly above $\beta \approx 0.06$ ( $6 \%$ speed of light). As shown in Fig. $5 \mathrm{c}$, the values of $\beta$ are in the range from $\approx 0.027(2.7 \%$ of $\mathrm{c})$ to $\approx 0.084(8.4 \%$ of $\mathrm{c})$ for ${ }^{12} \mathrm{C}$, and from $\approx 0.014(1.4 \%$ of $\mathrm{c})$ to $\approx 0.053(5.3 \%$ of $\mathrm{c}$ ) for ${ }^{16} \mathrm{O}$. Therefore, no significant ICF contribution is expected at the given values of $v_{r e l}$. However, the results presented in Fig.5c clearly demonstrate the onset of ICF at relatively lower value of $v_{\text {rel }}$ i.e, $\approx 0.027\left(\mathrm{~F}_{I C F} \approx 7 \%\right)$ in ${ }^{12} \mathrm{C}+{ }^{169} \mathrm{Tm}$ system, and at $\approx 0.014\left(\mathrm{~F}_{I C F} \approx 10 \%\right)$ in ${ }^{16} \mathrm{O}+{ }^{169} \mathrm{Tm}$ system. In both cases, the observed value of $\mathrm{F}_{I C F}$ is significant at well below the proposed onset value of $\beta$ (i.e., $6 \%$ of c). As such, it can be inferred that the ICF starts competing with $\mathrm{CF}$ even at slightly above barrier energies.

Further, as shown in Fig.5c, the value of $\mathrm{F}_{I C F}$ for ${ }^{12} \mathrm{C}$-projectile is lower than ${ }^{16} \mathrm{O}$-projectile for the entire measured energy range. The difference in $\mathrm{F}_{I C F}$ for two systems $\left({ }^{12} \mathrm{C},{ }^{16} \mathrm{O}+{ }^{169} \mathrm{Tm}\right)$ can be seen clearly, which indicates the dependence of $\mathrm{F}_{I C F}$ on projectile and/or on 


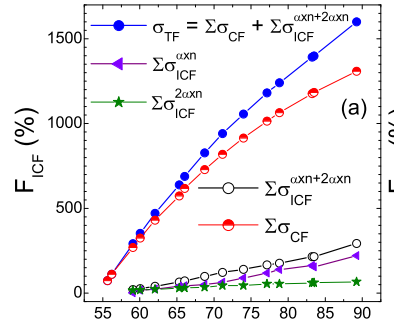

$\mathrm{E}_{\mathrm{lab}}$
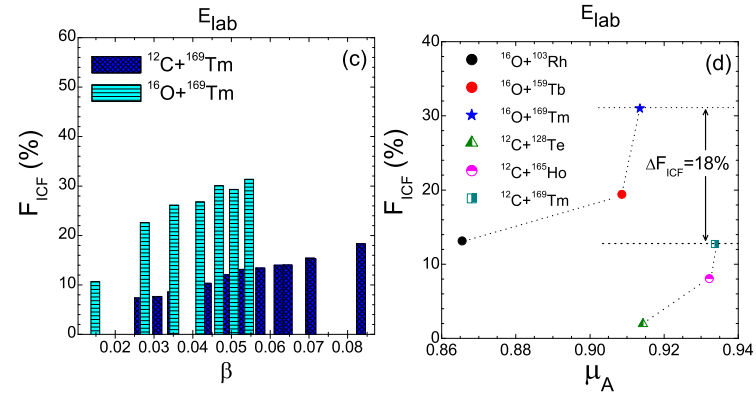

Fig. 5. (a) Total fusion cross-section $\left(\sigma_{T F}=\Sigma \sigma_{C F}+\Sigma \sigma_{I C F}\right)$ along with the sum of all CF-channels $\left(\Sigma \sigma_{C F}\right)$ and ICF-channels $\left(\Sigma \sigma_{I C F}\right)$ as a function of projectile energy, $(b)$ the ICF strength function for ${ }^{12} \mathrm{C}+{ }^{169} \mathrm{Tm}$ system (see text for description), (c) the value of $\mathrm{F}_{I C F}$ as a function of relative velocity $\left(\mathrm{v}_{r e l} \rightarrow \beta\right)$ for ${ }^{12} \mathrm{C},{ }^{16} \mathrm{O}+{ }^{169} \mathrm{Tm}$ systems, and $(b)$ the values of $\mathrm{F}_{I C F}$ obtained for ${ }^{12} \mathrm{C}+{ }^{128} \mathrm{Te},{ }^{165} \mathrm{Ho},{ }^{169} \mathrm{Tm}$ and ${ }^{16} \mathrm{O}+{ }^{103} \mathrm{Rh},{ }^{159} \mathrm{~Tb},{ }^{169} \mathrm{Tm}$ systems as a function of entrance channel mass asymmetry $\left(\mu_{A}\right)$ at a constant value of $\beta=0.053$. The dotted lines drawn through the data points guide the eyes for individual $\left({ }^{12} \mathrm{C}\right.$ and $\left.{ }^{16} \mathrm{O}\right)$ projectiles.

mass-asymmetry $\left(\mu_{A}\right)$ of interacting partners. In order to refine this e ffect, the values of $F_{C F}$ for nearby systems $\left({ }^{12} \mathrm{C}+{ }^{128} \mathrm{Te},{ }^{165} \mathrm{Ho},{ }^{169} \mathrm{Tm}\right.$ and $\left.{ }^{16} \mathrm{O}+{ }^{103} \mathrm{Rh},{ }^{159} \mathrm{~Tb},{ }^{169} \mathrm{Tm}\right)$ are deduced at a constant $\beta$ value (i.e., $\beta=0.053$ ), and plotted as a function of $\mu_{A}$ in Fig.5d. As shown in this figure, the Morgenstern's systematics do not explain the variation of $\mathrm{F}_{I C F}$ with $\mu_{A}$ for given projectile-target combinations. However, the value of $\mathrm{F}_{I C F}$ increases with $\mu_{A}$ for individual projectiles $\left({ }^{16} \mathrm{O}\right.$ and $\left.{ }^{12} \mathrm{C}\right)$. It is interesting to note that the ${ }^{12} \mathrm{C}+{ }^{169} \mathrm{Tm}$ system is a more mass asymmetric $\left(\mu_{A}=0.9337\right)$ system than ${ }^{16} \mathrm{O}+{ }^{169} \mathrm{Tm}$ system $\left(\mu_{A}=\right.$ $0.9135)$, but the value of $\mathrm{F}_{I C F}$ is $18.3 \%$ higher than that observed for ${ }^{12} \mathrm{C}+{ }^{169} \mathrm{Tm}$ system. The experimental data presented in Fig.5d for six projectile-target combinations clearly demonstrate strong projectile structure dependence of ICF fraction.

\section{Evidence of partial linear momentum transfer events in forward recoil ranges}

In previous sections, reaction dependent entry state spinpopulations and fraction of ICF in the production of $\alpha$ emitting channels are briefly discussed. These measurements conclusively demonstrate the onset of ICF at slightly above barrier energies in non-central interactions. However, for an additional evidence of fusion incompleteness at these energies and to validate data reduction procedure to deduce $\mathrm{F}_{I C F}$, the forward ranges (FRs) of heavy recoils have been measured for the same projectile-target combinations $\left({ }^{12} \mathrm{C},{ }^{16} \mathrm{O}+{ }^{169} \mathrm{Tm}\right)[5,8]$. The fact that the FRs of heavy recoils depend upon degree of linear momentum transfer $\left(\rho_{L M T}\right)$ from projectile to target nucleus. For ICF composites, where only a part of projectile fuses with target nucleus, the $\rho_{L M T}$ may be given as;

$$
\rho_{L M T}=\frac{P_{\text {frac }}}{P_{\text {proj }}}
$$

Here; $P_{\text {frac }}$ and $P_{\text {proj }}$ are the fractional and entire linear momentum of projectile, respectively. According to the range-energy formulation, entire LMT (i.e., $\rho_{L M T}=1$ ) from projectile to target nucleus in a CF reaction supposed to give maximum recoil velocity to the composite system $\left(\mathrm{CF} \rightarrow \mathrm{A}_{C N}=\mathrm{A}_{\text {target }}+\mathrm{A}_{\text {proj }}\right)$, and relatively less recoil velocity to the incompletely fused composite (ICF $\rightarrow$ $\mathrm{A}_{C N^{*}}=\mathrm{A}_{T}+\mathrm{A}_{\text {proj-fract }}$. . Consequently, the radio-nuclides populated via small $\rho_{L M T}$ are expected to show relatively smaller penetration depth in the stopping medium as compared to the entire LMT populations. The CF and ICF products can be disentangled from the analysis of FRRs of heavy recoils in in the framework of breakup fusion (BUF) model [?]. In this model, the projectile is assumed to breakup into constituent $\alpha$-clusters (e.g., ${ }^{16} \mathrm{O}$ may break-up into ${ }^{12} \mathrm{C}+\alpha$, and/or ${ }^{8} \mathrm{Be}+{ }^{8} \mathrm{Be}$ clusters) within the nuclear field of the target nucleus. Either fragments may get fuses with the target nucleus, and the remnant goes on moving in the the forward cone as a spectator

For the measurement of FRs of heavy recoils, the same experimental setup and technique as that of ICF strength function have been used. Detailed discussion on the methodology can be found in our recent papers $[5,8]$. However, some of the important steps to deduce the distributions of FRs of heavy recoils are given here. The irradiations were carried out in GPSC using energy-degradation activation technique followed by offline- $\gamma$-spectroscopy. A stack of thin Al-recoil-velocity filters of thicknesses varying from $\approx 10-45 \mu \mathrm{g} / \mathrm{cm}^{2}$ (sufficient to stop entire LMT events) has been placed downstream of the target foil, so that the recoiling CF and/or ICF products can be stopped in the stopping medium at different penetration depths. Keeping in mind the half-lives of interest, the irradiations were carried out for $\approx 10 \mathrm{hrs}$ duration for each incident energy. After the irradiation, the Al-recoil-velocity filters were taken out from the scattering chamber and counted separately using two pre-calibrated, high resolution HPGedetectors of 100 c.c. active volume. The HPGe-detectors were pre-calibrated for energy and efficiency using standard $\gamma$-sources (e.g., ${ }^{60} \mathrm{Co},{ }^{133} \mathrm{Ba}$ and ${ }^{152} \mathrm{Eu}$ ). The resolution of $\gamma$-detector was found to be $\approx 2.4 \mathrm{keV}$, for $1.33 \mathrm{MeV}$ $\gamma$-ray of ${ }^{60} \mathrm{Co}$. The residues were identified by their characteristic $\gamma$-lines, and confirmed by the decay-curve analysis. The induced activities can be used to measure the production probability of the reaction products. The measured intensities of the characteristic $\gamma$-lines have been used to determine the production cross-sections of the residues in each catcher foil using standard formulation [5]. The overall errors from all possible factors (for detail see ref. [8]) including the statistical error are estimated to be $\leq 13 \%$. The normalized yield of reaction products have been plotted as a function of cumulative catcher foil thicknesses to generate the distribution of FRs of heavy recoils. The distribution of FRs of heavy recoils have been fitted with 

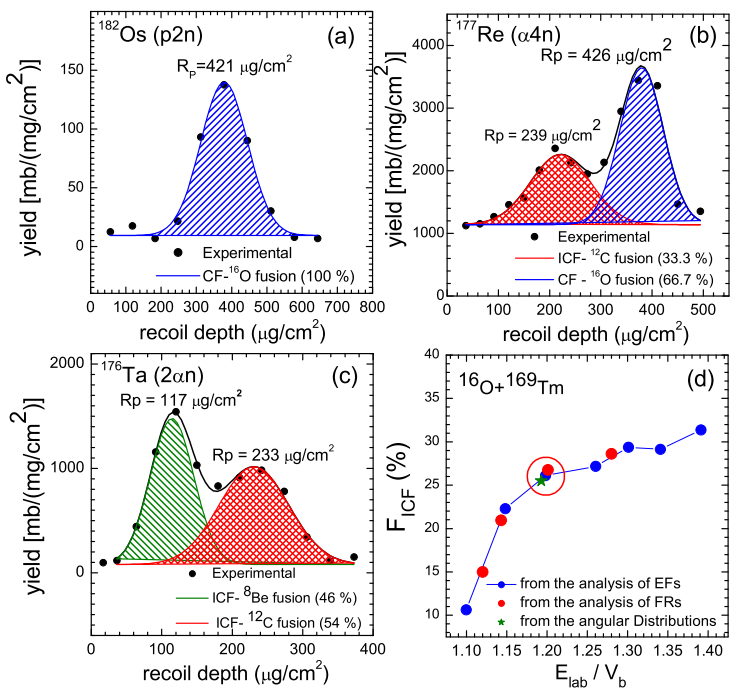

Fig. 6. The experimentally measured distributions of forward recoil ranges for $(a){ }^{182} \mathrm{Os}(\mathrm{p} 2 \mathrm{n}),(b){ }^{177} \mathrm{Re}(\alpha 4 \mathrm{n})$, and $(c){ }^{176} \mathrm{Ta}(2 \alpha \mathrm{n})$ residues produced in ${ }^{16} \mathrm{O}+{ }^{169} \mathrm{Tm}$ system at $\approx 87 \mathrm{MeV}$. $(d)$ The value of ICF fraction obtained from different approaches is compared to check the self consistency of three methods. Red circle around the data points shows the high degree of mutual agreement between different approaches with in the experimental uncertainties.

the Gaussian function for better analytical representation of data, and to deduce the value of most probable recoil ranges $\left(\mathrm{R}_{p(\text { exp })}\right)$ in the stopping medium $[5,8]$.

Fig.6(a) shows the distribution of FRs for ${ }^{182} \mathrm{Os}(\mathrm{p} 2 \mathrm{n})$ populated in ${ }^{16} \mathrm{O}+{ }^{169} \mathrm{Tm}$ system at $\approx 87 \mathrm{MeV}$. As shown in this figures, the data can be fitted by a Gaussian peak ( $P(\exp )$ $\approx 421 \mu \mathrm{g} / \mathrm{cm}^{2}$ ) indicating single LMT-component involved in the production of this residue. Fig.6(b-c) show the distribution of FRs for ${ }^{177} \operatorname{Re}(\alpha 4 \mathrm{n})$ and ${ }^{176} \mathrm{Ta}(2 \alpha \mathrm{n})$ residues. As shown in these figures, the distributions of FRs can be resolved into two Gaussian peaks, revealing the presence of more than one LMT-components associated with the fusion of ${ }^{16} \mathrm{O},{ }^{12} \mathrm{C}$ and/or ${ }^{8} \mathrm{Be}$ with the target nucleus. Refer to Fig.6b, the FRR distribution of residue ${ }^{179} \operatorname{Re}(\alpha 2 \mathrm{n})$ shows two LMT components at $\mathrm{R}_{P(\text { exp })} \approx 426 \mu \mathrm{g} / \mathrm{cm}^{2}$ (due to ${ }^{16} \mathrm{O}$-fusion) and at $\mathrm{R}_{P(\text { exp })} \approx 239 \mu \mathrm{g} / \mathrm{cm}^{2}\left({ }^{12} \mathrm{C}\right.$-fusion, where an $\alpha$ behaves as spectator). On the basis of observed LMT components at different penetration depth in the stopping medium $\mathrm{R}_{P(\text { exp })}$, it can be inferred that the residues ${ }^{177} \operatorname{Re}(\alpha 4 \mathrm{n})$ is populated via both CF and ICF processes. The population of ${ }^{177} \operatorname{Re}(\alpha 4 \mathrm{n})$ via both $\mathrm{CF}$ and/or ICF can be represented as;

(i) $\mathrm{CF}^{-16} \mathrm{O}$ :

$$
{ }^{16} \mathrm{O}+{ }^{169} \mathrm{Tm} \Rightarrow{ }^{185} \mathrm{Ir}^{\star} \Rightarrow{ }^{177} \mathrm{Re}+\alpha 4 \mathrm{n}(\text { from eq-CN})
$$

(ii) $\mathrm{ICF}^{12} \mathrm{C}$ :

$$
\begin{gathered}
{ }^{16} \mathrm{O}\left({ }^{12} \mathrm{C}+\alpha\right) \Rightarrow{ }^{12} \mathrm{C}+{ }^{169} \mathrm{Tm} \Rightarrow{ }^{181} \mathrm{Re}^{\star} \Rightarrow{ }^{177} \mathrm{Re}+4 \mathrm{n} \\
\text { (from eq-CN) }+\alpha \\
\text { (one direct- } \alpha \text { as spectator) }
\end{gathered}
$$

Similarly, the FRR distribution of ${ }^{176} \mathrm{Ta}(2 \alpha \mathrm{n})$ shows two LMT components at the mean penetration depth
$\mathrm{R}_{P(\exp )} \approx 233 \mu \mathrm{g} / \mathrm{cm}^{2}$ due to ${ }^{12} \mathrm{C}$-fusion, and at $\mathrm{R}_{P(\exp )} \approx$ $117 \mu \mathrm{g} / \mathrm{cm}^{2}$ due to ${ }^{8} \mathrm{~B}$ and/or $2 \alpha$ (in successive mode)fusion with target nucleus. The observation of two LMT components at relatively smaller penetration depth than that observed for ${ }^{16} \mathrm{O}$-fusion (i.e., $\mathrm{R}_{P(\exp )} \approx 421 \mu \mathrm{g} / \mathrm{cm}^{2}$ ) indicates the production of ${ }^{176} \mathrm{Ta}(2 \alpha \mathrm{n})$ residue only via ICF. The production of ${ }^{176} \mathrm{Ta}(2 \alpha \mathrm{n})$ only via ICF can be explained as;

(i) $\mathrm{ICF}^{12} \mathrm{C}$ :

$$
\begin{gathered}
{ }^{16} \mathrm{O}\left({ }^{12} \mathrm{C}+\alpha\right) \Rightarrow{ }^{12} \mathrm{C}+{ }^{169} \mathrm{Tm} \Rightarrow{ }^{181} \mathrm{Re}^{\star} \Rightarrow{ }^{176} \mathrm{Ta}+\alpha \mathrm{n} \\
\text { (from eq- } \mathrm{CN} \text { ') }+\alpha \\
\text { (one direct- } \alpha \text { as spectator) }
\end{gathered}
$$

(ii) $\mathrm{ICF}^{-}{ }^{8} \mathrm{Be}$ :

$$
\begin{gathered}
{ }^{16} \mathrm{O}\left({ }^{8} \mathrm{Be}+{ }^{8} \mathrm{Be} \text { or } 2 \alpha\right) \Rightarrow{ }^{8} \mathrm{Be}+{ }^{169} \mathrm{Tm} \Rightarrow{ }^{177} \mathrm{Ta}^{\star} \Rightarrow{ }^{176} \mathrm{Ta} \\
+\mathrm{n} \text { (from eq-CN') }+2 \alpha \\
\text { (two direct- } \alpha \text { as spectator) }
\end{gathered}
$$

The value of $\mathrm{R}_{P}^{\text {exp }}$ for different residues have also been compared with $\mathrm{R}_{P}^{\text {the }}$ of ERs estimated by adopting breakup-fusion description of massive transfer reactions given by Udagawa and Tamura [29]. The values of $\mathrm{R}_{P}^{\text {exp }}$ are found to be in good agreement with theoretical ones.

In order to validate the data reduction procedure used to deduce ICF fraction in an $\alpha$-emitting channel, the relative contributions of $\mathrm{CF}$ and ICF have been accounted and translated into $\mathrm{F}_{I C F}$. The value of $\mathrm{F}_{I C F}$ deduced from three different techniques, namely: $(i)$ from the analysis of FRRs, (ii) angular distributions, and (iii) from the analysis of EFs, have been plotted as a function of $\mathrm{E}_{\text {beam }} / \mathrm{CB}$ in Fig. $5 \mathrm{~d}$, where $\mathrm{E}_{\text {beam }}$ is the beam energy and $\mathrm{CB}$ is the Coulomb barrier for the ${ }^{16} \mathrm{O}+{ }^{169} \mathrm{Tm}$ system. Solid line through the data points is drawn to guide the eyes. As shown this figure, the value of $\mathrm{F}_{I C F}$ deduced from three approaches at a constant normalized incident energy $\left(\mathrm{E}_{\text {beam }} / \mathrm{CB}=1.2\right)$ is found to be in good mutual agreement within the experimental uncertainties. This puts faith in our measurements and data reduction procedure.

\section{Summary}

In this paper, recent experimental results on low energy ICF are presented. In order to probe associated $\ell$ values in the production of different reaction channels, the spin-distributions and feeding intensity profiles of ERs have been measured in ${ }^{12} \mathrm{C},{ }^{16} \mathrm{O}+{ }^{169} \mathrm{Tm}$ systems at near and above barrier energies. The spin-distributions of ICF$\alpha \times n / 2 \alpha \times n$-channels are found be distinctly different than that observed for $\mathrm{CF}-\mathrm{xn} / \mathrm{pxn} / \alpha \mathrm{xn}$-channels. This indicates reaction dependent entry state spin-populations. The $\mathrm{CF}$ products are found to strongly fed over a broad spin range towards the band head. However, the spin-distribution(s) associated with ICF are found to be arrised from the narrow spin population, localized near and/or above to the critical angular momentum for CF. The trend of spindistributions indicates the competition from successively opened ICF channels for each value of $\ell$ above $\ell_{\text {crit }}$ for normal fusion $(\mathrm{CF})$. The population of low spin states are found to be hindered, and/or less fed in case of ICF channels. At slightly above barrier energies, the ICF is found to be originated from non-central interactions $\left(\ell \geq \ell_{\text {crit }}\right)$ due 
to the influence of centrifugal potential, where the limit of driving angular momenta do not allow CF. The direct $\alpha$-multiplicity increases in forward cone with input $<\ell>$ values at a particular projectile energy. For an example, at $\approx 5.6 \mathrm{AMeV}$ the value of $\langle\ell\rangle$ for $\mathrm{CF}-\mathrm{xn} / \mathrm{pxn}-\mathrm{B} / \alpha \mathrm{xn}-\mathrm{B}$, and ICF- $\alpha$ xn-F, and ICF- $2 \alpha \mathrm{xn}-\mathrm{F}$ channels are found to be $\approx 7.5 \hbar, \approx 10 \hbar$ and $\approx 13.5 \hbar$, respectively. This shows that the high $\ell$-values associated with non-central interactions essentially contribute to open up ICF (direct- $\alpha$-emitting)channels. Further, the comparison of $\ell$-values involved in $\mathrm{CF}$ and ICF residues populated via $\mathrm{xn} / \mathrm{pxn} / \alpha \mathrm{xn}-\mathrm{B} / \alpha \mathrm{xn}-\mathrm{F}$ channels give direct evidence to populate high spin states in final reaction products via ICF, which is not possible to achieve via $\mathrm{CF}$ at a given projectile energy.

The findings of spin-distribution measurement are confirmed in a complementary experiment, where ICF strength function is deduced from the analysis of experimental EFs in the framework of statistical model code PACE4. In order to get insights into the onset and strength of ICF, the value of $\mathrm{F}_{I C F}$ has been deduced as a function of projectile energy (i.e., from $1.02 \mathrm{~V}_{b}$ to $1.64 \mathrm{~V}_{b}$ ) and entrance channel mass-asymmetry. The value of $\mathrm{F}_{I C F}$ increases from $\approx 7 \%$ to $\approx 18 \%$ with in the studied energy range. The results presented in section- 3 clearly demonstrate the onset of ICF at the value of $\beta \approx 0.027\left(\mathrm{~F}_{I C F}\right.$ $\approx 7 \%$ ) in ${ }^{12} \mathrm{C}+{ }^{169} \mathrm{Tm}$ system, and at the value of $\beta \approx$ $0.014\left(\mathrm{~F}_{I C F} \approx 10 \%\right)$ in ${ }^{16} \mathrm{O}+{ }^{169} \mathrm{Tm}$ system. In both cases, the observed value of $\mathrm{F}_{I C F}$ is significant at well below the proposed onset $\beta$-value (i.e., $6 \%$ of c) proposed by Morgenstern et al., [31-33]. A comparison of $\mathrm{F}_{I C F}$ for ${ }^{12} \mathrm{C}+{ }^{169} \mathrm{Tm}$ and ${ }^{16} \mathrm{O}+{ }^{169} \mathrm{Tm}$ systems displays higher ICF probability for ${ }^{16} \mathrm{O}+{ }^{169} \mathrm{Tm}$ system for the entire measured energy range. This shows strong projectile structure effect on ICF fraction. The existence of ICF at well below the proposed onset $\beta$-value supplements Morgenstern's massasymmetry systematics.

Further, the data reduction procedure used to account the ICF fraction in an $\alpha$-emitting channel is validated from the measurement and analysis of forward recoil ranges. It has been found that the $\alpha$-emitting channels are populated via both $\mathrm{CF}$ and ICF processes. The forward recoil ranges are analyzed on the basis of breakup fusion model description of massive transfer reaction given by Udagawa and Tamura [29]. More than one linear momentum transfer (LMT) components attributed to the full (fusion of ${ }^{16} \mathrm{O}$ ) and/or partial fusion $\left({ }^{12} \mathrm{C}\right.$ and/or ${ }^{8} \mathrm{Be}$ fusion from ${ }^{16} \mathrm{O}$ ) of projectile have been observed. The values of $\mathrm{R}_{P(\text { exp })}$ are found to be consistent with the theoretical predictions within the experimental uncertainties. The value of $\mathrm{F}_{I C F}$ obtained from the analysis of forward recoil ranges is found to be in good agreement with that deduced from the measurement and analysis of EFs. The findings of these experiments clearly demonstrate; $(a)$ the reaction dependent decay patterns, $(b)$ the origin of low energy ICF events from non-central interactions, $(c)$ the possibility to selectively populate high spin states via ICF, $(d)$ the existence of ICF at slightly above barrier energies, and $(e)$ strong projectile structure effect on ICF fraction. As such, it can be inferred that the ICF is a process of greater importance even at low projectile energies, where CF supposed to be the sole contributor. However, it would be interesting to explore ICF dynamics at low incident energies using non- $\alpha$-cluster beams (e.g., ${ }^{13} \mathrm{C},{ }^{14} \mathrm{~N}$ and ${ }^{18} \mathrm{O}$ ) with ${ }^{169} \mathrm{Tm}$ target nucleus.

\section{Acknowledgments}

The authors wish to acknowledge that the work presented in this paper is the outcome of many experiments performed at the Inter-University Accelerator Center (IUAC), New Delhi by AMU-IUAC collaboration. We thank all our collaborators for their help during the experiments and very useful discussions, and Prof. Amit Roy for motivating us to carry out these measurements. One of the authors P.P.S. thanks to GSI-Helmholtz Centre for Heavy Ion Research GmbH and Technical University Darmstadt, Germany for financial support. R. P. \& B. P. S. acknowledge the research grants received from Department of Science and Technology (DST) and University Grant Commission (UGC), Government of India.

\section{References}

1. Pushpendra P. Singh et al., Phys. Lett. B671, 20 (2009), and the references therein.

2. Pushpendra P. Singh et al., Phys. Rev. C80, 064603 (2009)

3. Pushpendra P. Singh et al., Phys. Rev. C78, 017602 (2008), and the references therein.

4. Pushpendra P. Singh et al., Phys. Rev. C77, 014607 (2008).

5. Pushpendra P. Singh et al., Eur. Phys. J. A 34, 29 (2007), and the refences there in.

6. Pushpendra P. Singh et al., Phys. Lett. B (to be submitted)

7. Devendra P. Singh et al., Phys. Rev. C81, 054607 (2010).

8. Unnati Gupta et al., Phys. Rev. C80, 024613 (2009).

9. Unnati Gupta et al., Nucl. Phys. A811, 77 (2008).

10. Pushpendra P. Singh et al., Jour. of Phys: Conf. Sr. 282, 012019 (2011).

11. Pushpendra P. Singh et al., Eur. Phys. J. Web. of Conf. 2, 10004 (2010).

12. D. J. Hinde and M. Dasgupta, Phys. Rev. C 81, 064611 (2010), and the references therein.

13. A. Diaz-Torres, Phys. Rev. Lett. 98, 152701 (2007), and the references therein.

14. P. R. S. Gomes et al., Phys. Lett. B601, 20 (2004).

15. P. R. S. Gomes et al., Phys. Rev. C73, 064606 (2006).

16. E. Z. Buthelezi et al., Nucl. Phys. A734, 553 (2004).

17. L. R. Gasques et al., Phys. Rev. C79, 034605 (2009).

18. L. R. Gasques et al., Phys. Rev. C74, 064615 (2006).

19. P. E. Hodgson, E. Gadioli and E. Gadioli Erba, Introductory Nuclear Physics, Chapter 23, Clarendon Press, Oxford (1997).

20. J. Wilczynski et al., Phys. Rev. Lett. 45, 606 (1980).

21. J. Wilczynski et al., Nucl. Phys. A 373, 109 (1982).

22. K. Siwek-Wilczynska et al., Phys. Rev. Lett. 42, 1599 (1979).

23. H. C. Britt and A. R. Quinton, Phys. Rev. 124, 877 (1961).

24. J. Galin et al., Phys. Rev. C 9, 1126 (1974).

25. W. Trautmann et al., Phys. Rev. Lett. B 53, 1630 (1984). 
26. T. Inamura et al., Phys. Lett. 68B, 51 (1977).

27. T. Inamura et al., Phys. Lett. 84B, 71 (1982).

28. T. Inamura et al., Phys. Rev. C 32, 1539 (1985).

29. T. Udagawa and T. Tamura, Phys. Rev. Lett. 45, 1311 (1980).

30. J. P. Bondrof et al., Nucl. Phys. A 333, 285 (1980).

31. H. Morgenstern et al., Z. Phys. A313, 39 (1983).

32. H. Morgenstern et al., Phys. Rev. Lett. 52, 1104 (1984).

33. H. Morgenstern et al., Z. Phys. A324, 443 (1986).

34. C. Gerschel, Nucl. Phys. A 387, 297 (1982), and the references there in.

35. A. Garvon, Phys. Rev. C21, 230 (1980). 